\title{
DERNİ̀RE HEURE
}

\section{Reprogrammation nucléaire d'une cellule différenciée On efface tout et on recommence}

Laure Coulombel

> La communauté scientifique, récemment échaudée, avait accueilli avec prudence l'annonce par une équipe japonaise dans Cell en août 2006, d'une possible reprogrammation, chez la souris, de cellules somatiques adultes en cellules ayant certaines des caractéristiques de cellules souches embryonnaires (dénommées iPS, induced pluripotent stem) par l'expression forcée de 4 facteurs de transcription $[1,2]$. La confirmation éclatante de ces données par deux équipes de Harvard (États-Unis), celle de R. Jaenisch [3], et celle de K. Hochedlinger [4], vient d'être publiée dans Nature, dans le premier numéro de Cell Stem Cell, un nouveau journal édité par CellPress; un second article de l'équipe japonaise de S. Yamanaka les accompagne dans Nature également [5]. On peut donc maintenant donner libre cours à notre enthousiasme, car cette découverte fera date et les conséquences sur le plan conceptuel et médical seront sans doute considérables s'il s'avère que les observations peuvent être dupliquées chez l'homme.

La nouveauté ne réside pas dans la possibilité de conférer à un noyau différencié, et donc restreint dans la palette de gènes exprimés, l'organisation épigénomique et les compétences d'un noyau pluripotent, mais dans la simplicité de cette démarche. On sait le faire par transfert nucléaire d'un noyau somatique dans un ovocyte énucléé $[6,7]$, ou par la fusion d'une cellule somatique avec une cellule embryonnaire, comme l'ont montré plus récemment les équipes de K. Eggan [8] ou A. Smith [9], qui, en 2005 , prédisaient déjà la simplification du processus de reprogrammation de noyaux somatiques vers un état embryonnaire pluripotent. Mais ici, noyau et cytoplasme proviennent de la même cellule.

\section{Quatre petits facteurs de transcription et puis s'en vont...}

K. Takahashi et S. Yamanaka, dans leur article d'août 2006 [1], avaient testé de multiples combinaisons de facteurs pour finalement déterminer que l'expression de 4 ADNc codant pour quatre facteurs de transcription, Sox2, oct-4, c-myc et klf4 - les 2 premiers sont indispensables au maintien d'un état pluripotent - conférait à des fibroblastes embryonnaires de souris (MEF) mais aussi à des fibroblastes de la queue de souris (TTF) adulte quelques unes des propriétés de cellules souches embryonnaires. Mais ces cellules iPS étaient un peu «bâtardes»: les profils transcriptionnel et de méthylation différaient de ceux de cellules ES établies, et la contribution des iPS au développement embryonnaire après leur transfert dans un blastocyste secondairement réimplanté, était très limitée [1].

$\varepsilon$ n moins d'un an, ces restrictions ont été résolues...

Si les quatre facteurs de transcription sont toujours les mêmes (et le quatuor
L. Coulombel : Médecine/Sciences et Inserm U602, Hôpital Paul Brousse, 94817 Villejuif Cedex, France. Icoulombel@medecinesciences.org

est indissociable), la procédure de sélection des cellules iPS a été affinée: elles est fondée sur l'expression d'un gène rapporteur placé sous le contrôle des promoteurs des gènes endogènes 0 ct-4 ou Nanog [3, 4], normalement éteints dans les fibroblastes, et dont la réactivation est le meilleur témoin d'une reprogrammation de type embryonnaire, puisque 0 ct-4, nanog et Sox2 représentent la «triade» nécessaire à l'état pluripotent, in vivo comme in vitro. La stratégie initiale des Japonais en 2006 consistait à utiliser l'expression du gène $f b x 15$, certes une cible de oct-4, mais dont l'expression n'est pas nécessaire au maintien de l'état pluripotent des cellules souches. Ce choix explique probablement qu'aient été sélectionnées des cellules iPS probablement non pluripotentes [1].

\section{Le fabuleux destin d'un banal fibroblaste de la queue de souris}

Parmi les MEF ayant intégré les quatre transgènes (50\%), l'efficacité de reprogrammation en cellules iPS est de l'ordre de 0,05\%, et le processus prend 3 semaines. Les cellules iPS prolifèrent sous forme de colonies compactes, comme le font les cellules $\varepsilon S$, et, comme elles, requièrent l'ajout de LIF (leukemia inhibiting factor), mais peuvent se passer de sous-couches fibroblastiques.

Injectées sous la peau de souris immunodéficientes, les cellules iPS forment 
des tératomes, contenant les dérivés des trois feuillets embryonnaires, ce qui indique leur pluripotence, et manifestement, la différenciation mésodermique et ectodermique des iPS peut être induite in vitro, mais, dans les 3 articles, l'accent est surtout mis sur les expériences in vivo. En effet, la preuve irréfutable de la pluripotence de cellules $\varepsilon S$ est la démonstration de leur participation in vivo à l'ensemble des tissus au cours du développement embryonnaire après leur injection dans un blastocyste ensuite réimplanté dans un utérus gravide. Les cellules iPS ont cette capacité : 7 sur 15-20 clones pour les japonais, environ $10 \%$ des blastocystes injectés $(n=365)$ pour les Américains, donnent naissance à des animaux chimères viables, le taux de chimérisme variant de 5 à $70 \%$. Une contribution à la lignée germinale a aussi été prouvée car, après accouplement de certains des mâles chimériques $F 1$ à des femelles normales, la présence du génotype 0ct-4- ou Nanog-iPS a été trouvée dans la proportion attendue des embryons ou des souriceaux F2

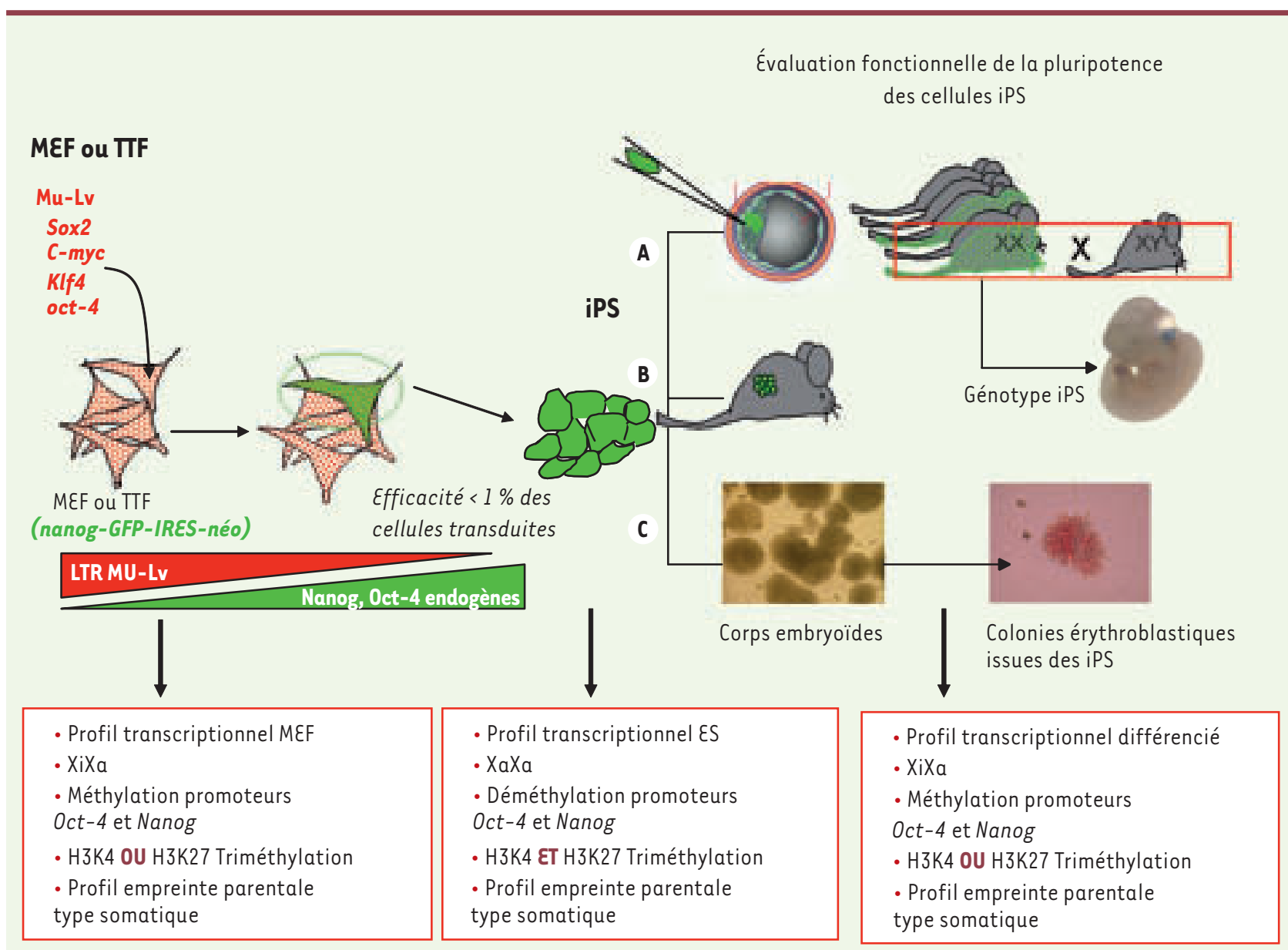

Figure 1. Stratégie expérimentale utilisée pour « reprogrammer 》 des fibroblastes différenciés en cellules souches pluripotentes. Les fibroblastes (MEF, murine embryonic fibroblasts, ou TTF, tip tail fibroblasts) sont préparés à partir de souris transgéniques dont le promoteur Nanog ou 0ct4 endogène contrôle l'expression de la GFP. Ces fibroblastes sont transduits via des rétrovirus Moloney contenant 4 ADNc codant pour sox2, oct4, cmyc et klf4. Les cellules ayant réactivé les gènes Oct4 ou Nanog endogènes sont sélectionnées sur l'expression de la GFP 15-21 jours après la transduction. Certaines prolifèrent sous la forme de colonies de cellules adhérentes, exprimant plusieurs marqueurs caractéristiques de cellules ES (SSEA-1, TRA...) et sont appelées iPS pour induced pluripotent stem cells. La pluripotence fonctionnelle de ces cellules est attestée par (A) leur capacité de contribuer à des animaux chimères lorsqu'elles sont injectées dans un blastocyste; certains mâles chimériques ont été accouplés à des femelles sauvages et le génotype des embryons F2 analysé; il est de type iPS dans la proportion attendue, prouvant la transmission germinale de la reprogrammation; $(B)$ de former des tératomes composés des dérivés des 3 feuillets embryonnaires lorsqu'elles sont injectées à des souris immunodéficientes; $(C)$ de se différencier in vitro en cellules mésodermiques (la différenciation en érythroblastes via l'étape intermédiaire de corps embryonnaires est illustrée ici), neurectodermiques ou encore endodermiques, en présence des inducteurs adéquats. Les trois encadrés indiquent les principales caractéristiques «épigénétiques » (méthylation de l'ADN, marques des histones, statut du chromosome X, et empreinte parentale), des MEF initiaux, des iPS pluripotentes, et des cellules différenciées issues des iPS. 
prouvant la différenciation des cellules iPS en cellules germinales fonctionnelles. L'agrégation de cellules iPS à des embryons tétraploïdes (test plus rigoureux car ces embryons tétraploïdes ne participent qu'à la formation des annexes, et l'embryon dérive entièrement des cellules iPS agrégées) a également abouti à la production d'embryons viables issus de cellules iPS.

On peut donc conclure que, sur des critères phénotypiques et fonctionnels rigoureux, les cellules iPS, issues de cellules différenciées fibroblastiques, ont acquis tous les attributs d'un état pluripotent. L'analyse transcriptionnelle élargie par microarray confirme aussi la proximité entre cellules $\varepsilon S$ et iPS, et les divergences profondes de ces deux types cellulaires avec les cellules fibroblastiques MEF ou TTF d'origine.

Il est intéressant de mentionner que dans ces cellules iPS, ce sont les seuls gènes Oct4 et Nanog qui assurent la pérennisation de l'état pluripotent. En effet, on sait depuis 20 ans que les régions enhancer du rétrovirus Moloney utilisé pour la transduction des ADNc, ne fonctionnent pas dans les cellules embryonnaires, contrairement aux cellules somatiques adultes. De fait, l'activité des LTR virales s'éteint rapidement lors de la reprogrammation des fibroblastes en iPS, ce qui indique que l'induction du processus de reprogrammation ne requiert que transitoirement l'expression des 4 facteurs exogènes, le relais étant rapidement pris par l'expression endogène de 0 ct4 et Nanog.

\section{À vos marques... épigénétiques}

L'extinction des LTR virales suggérait l'expression de novo de méthylases par les IPS, et donc la capacité de ces iPS de modifier leur environnement épigénétique. Le passage d'un programme nucléaire somatique - de potentialité restreinte - à un programme embryonnaire - pluripotent
- implique une modification de la méthylation de I'ADN ainsi que l'effacement des modifications des molécules d'histone associées à l'état différencié et l'acquisition d'un état dynamique de la chromatine caractéristique de l'état pluripotent [10]. On peut détecter ces modifications en analysant le profil de méthylation de I'ADN, celui des lysines K4 et K27 des histones $\mathrm{H} 3$ associées aux gènes cibles clé, l'état d'activation des gènes soumis à l'empreinte parentale, et la réactivation du chromosome $X$ inactif dans les cellules femelles. La fusion avec des cellules ES peut conduire à la réactivation de gènes comme 0ct4, et du chromosome $X$ inactif, mais elle ne permet pas celle des gènes soumis à l'empreinte ( $H 19$, meist, peg3, snrp) - ce processus requiert une fusion avec des cellules EG (embryonic gonad) derivées des cellules germinales primordiales (PGC). Les cellules iPS se comportent comme des cellules $E S$ et non pas comme des cellules $\varepsilon G$, et gardent l'empreinte parentale somatique.

L'analyse initiale des cellules fbx15-iPS montrait une reprogrammation épigénétique incomplète et anarchique: ainsi, les promoteurs des gènes 0ct4 et Nanog endogènes n'étaient que partiellement déméthylés. Mais ce n'est plus le cas des promoteurs 0ct-4 et Nanog des iPS décrites dans les deux articles de Nature et celui de Cell Stem Cell, qui sont complètement déméthylés, en accord avec un profil typique de cellules ES. Ces cellules résistent, comme le font les cellules $\varepsilon S$, et à la différence des cellules différenciées, à une inactivation de l'enzyme dnmtl ADN-méthyltransférase.

Les modifications de la structure chromatinienne des noyaux de cellules $\varepsilon S$ ont été récemment décrites, et nous en avons fait état dans Médecine/Sciences [11-13]. Une des particularités est la juxtaposition, sur certains nucléosomes, de marques chromatiniennes d'activation (triméthylation de la lysine 4 de l'histone H3, H3K4me3) et de répression (H3K27me3) au niveau des gènes cibles de Oct4 et Nanog, alors que dans les cellules somatiques différenciées, l'une ou l'autre de ces marques est présente selon que le gène est actif ou réprimé. Les expériences de ChIP à partir des cellules iPS montrent que les gènes cibles de oct4 et nanog portent les modifications bivalentes des histones $\mathrm{H} 3$.

Dernier argument confirmant l'acquisition d'un état épigénétique « embryonnaire » des IPS, les deux chromosomes $X$ sont actifs, comme en témoigne l'expression bi-allélique de gènes liés à I'X, et la régulation appropriée des trois ARN non codants contrôlant réciproquement l'inactivation/activation de I'X: une expression de Tsix, et l'absence de Xist et Xite [14]. L'induction par l'acide rétinoïque de la différenciation des iPS, qui leur fait perdre le statut pluripotent, comme elle le fait pour les cellules $\varepsilon S$, induit une inactivation d'un des chromosomes $X$, couvert par Xist et enrichi en zones méthylées. L'utilisation d'un transgène rapporteur lié à I'X ( $X$ GFP) a permis en outre de démontrer le caractère aléatoire de cette inactivation. Ainsi, les iPS semblent enclencher un processus normal de mise en place d'inactivation de l'X, un événement qui, normalement, n'a pas lieu en dehors du développement.

\section{عt maintenant ?}

Ces articles confirment l'extraordinaire capacité d'être reprogrammés des noyaux de nos cellules somatiques: finalement quatre gènes avec des rôles de type «facteur de transcription » ont la capacité de remettre en place un programme pluripotentiel, et la reprogrammation via la perte et le gain des marques épigénétiques appropriées accompagne ce processus d'activation génique. Ce quatuor de transgènes n'est requis que très transitoirement, les cellules iPS 
pérennisant de façon autonome leur état pluripotent. Selon toute probabilité, l'on pourra donc se dispenser à l'avenir de vecteurs rétroviraux, et leur substituer soit des adénovirus, qui seront ensuite dilués avec les divisions, soit peut-être des petites molécules activant les gènes clés endogènes, ce qui serait souhaitable dans une perspective médicale. Même si l'expression virale est éteinte dans les cellules iPS, c-Myc est réactivé et induit des tumeurs dans les cellules différenciées de $10 \%$ des animaux chimériques $\mathrm{Fl}$ [4]. Des deux obstacles à l'utilisation thérapeutique des cellules $\varepsilon S$, incompatibilité immunologique et risque tumoral, seul le second subsisterait dans le cas des cellules iPS. Mais peut-être n'est-il pas nécessaire de refaire tout le chemin jusqu'aux cellules embryonnaires et une reprogrammation en progéniteurs spécifiques d'un tissu pourraitelle être envisagée?

Tout n'est pas résolu, la stabilité génétique, épigénétique et fonctionnelle à long terme de ces cellules n'est pas connue, et sur un plan plus fondamental, le mystère demeure quant à la nature de la cellule cible initiale et aux mécanismes précis de cette reprogrammation. Existe-t-il d'autres combinaisons de gènes encore plus appropriées. Toute cellule différenciée a-t-elle ce potentiel ? Si c'est le cas, I'on devrait espérer améliorer l'efficacité du processus. La cellule ciblée par cette reprogrammation est-elle une cellule souche? Peut-on alors oser I'hypothèse que les cellules souches adultes décrites sous différents vocables (MAPC, etc.), et dont l'existence est incertaine, seraient l'équivalent spontané de ce processus ? Nous ne sommes encore que chez la souris... mais peut-être pas pour très longtemps. Onze ans après Dolly, ces observations fascinantes sont probablement un milestone aussi important. $\diamond$ How to teach an old fibroblast to do new tricks

\section{REMERCIEMENTS}

Je remercie Edith Heard, Pierre-Antoine Desfossez, Xavier Vignon et Pierre Savatier pour leur relecture critique et pour leurs suggestions qui ont permis l'amélioration de ce texte.

\section{RÉFÉRENCES}

1. Takahashi K, Yamanaka S. Induction of pluripotent stem cells from mouse embryonic and adult fibroblast cultures by defined factors. Cell 2006 ; $126: 663-76$.
2. Coulombel L. Des cellules en état de crise identitaire. Med Sci (Paris) 2007 ; $22: 835$.

3. Wernig M, Meissner A, Foreman $R$, et al. In vitro reprogramming of fibroblasts into a pluripotent EScell-like state. Nature 2007 ; 6 juin online.

4. Maherali N, Sridharan R, Xie W, et al. Directly reprogrammed fibroblasts show global epigenetic remodeling and widespread tissue contribution Cell Stem Cell 2007; $1:$ 55-70.

5. Okita K, Ichisaka T, Yamanaka S. Generation of germline-competent induced pluripotent stem cells. Nature 2007 ; 6 juin online.

6. Campbell KH, McWhir J, Ritchie WA, Wilmut I. Sheep cloned by nuclear transfer from a cultured cell line. Nature 1996 ; $380: 64-6$.

7. Yang X, Smith SL, Tian XC, et al. Nuclear reprogramming of cloned embryos and its implications for therapeutic cloning. Nat Genet $2007 ; 39: 295-302$

8. Cowan CA, Atienza J, Melton DA, Eggan K. Nuclear reprogramming of somatic cells after fusion with human embryonic stem cells. Science 2005; 309: 1369-73.

9. Silva J, Chambers I, Pollard S, Smith A. Nanog promotes transfer of pluripotency after cell fusion. Nature 2006 ; 441 : 997-1001.

10. Surani MA, Hayashi K, Hajkova P. Genetic and epigenetic regulators of pluripotency. Cell 2007 ; 128: 747-62.

11. Boyer LA, Plath K, Zeitlinger J, et al. Polycomb complexes repress developmental regulators in murine embryonic stem cells. Nature 2006 ; $441: 349-53$.

12. Szutorisz H, Dillon N, Tora L. Le protéasome limite la transcription dans les cellules souches embryonnaires. Med Sci (Paris) 2007 ; 23 : 351-3.

13. Negre N, Cavalli G. Polycomb maîtrise la destinée cellulaire. Med Sci (Paris) $2006 ; 22: 1033-5$.

14. Augui $S$, Heard $\varepsilon$. Le rendez-vous des chromosomes X. Med Sci (Paris). 2006 ; 22 : 910-1.

\section{TIRÉS À PART}

L. Coulombel

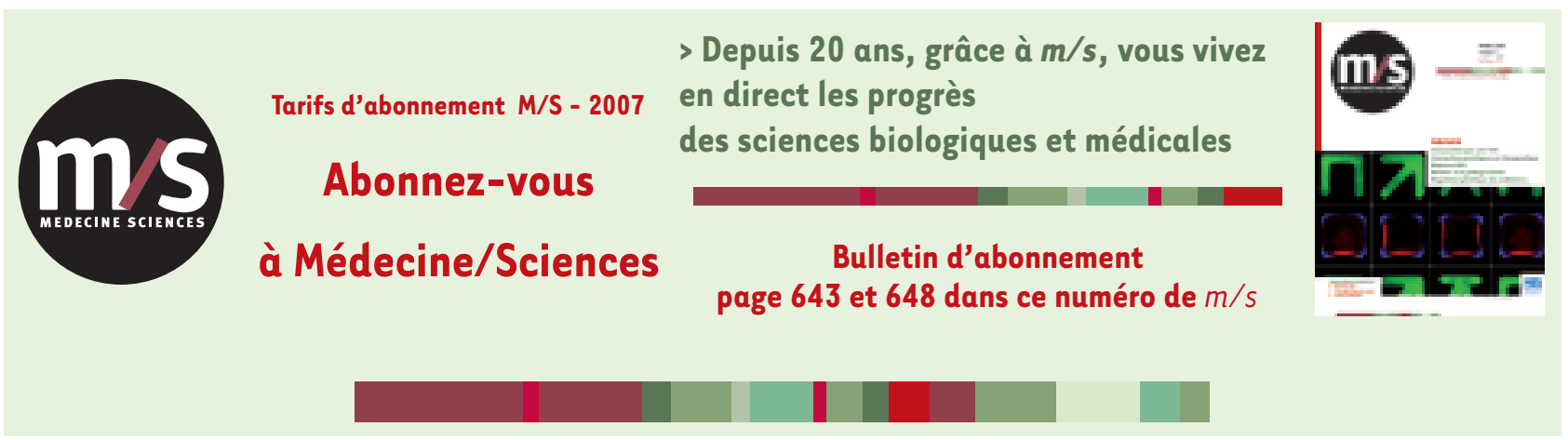

